\title{
Development and implementation of the GIT- modelling bioreactor system: the way to reducing a carbon footprint
}

\author{
Donskoy Danila ${ }^{1 *}$, Katin Oleg ${ }^{1}$ and Alekseenko Ludmila ${ }^{1}$ \\ ${ }^{1}$ Don State Technical University, Rostov-on-Don, 344000, Russia
}

\begin{abstract}
The purpose of this article is to analyze and compare the carbon footprint value between «in vivo» and «in vitro» microbiological experiments in chicken GIT. The SHIME-style bioreactor for modeling the processes occurring in the gastrointestinal tract of chickens is developed. For «in vitro» estimation - use analytic and experimental results, carrying out on developed chicken GIT model. For « in vivo» estimation, use the carbon footprint of two mounts old broiler chicken. Assessments showed that «in vitro» carbon footprint constitutes about $15 \%$ of «in vivo» one. The most significant contributors to «in vitro» carbon footprint are nutrient medium (80\%), then control computer (10\%), then heather $(5 \%)$. Recommendations for further carbon footprint reduction for «in vitro» experiments are formulated.
\end{abstract}

\section{Introduction}

At present, the shift of focus from experiments «in vivo» to «in vitro» is an established global trend in microbiology. Along with many ethical, microbiological, and other advantages, conducting a significant part of in vitro research allows reducing the carbon footprint formed during the development of micro- and prebiotic drugs by reducing the contribution from living organisms used in the process of experiments. This requirement is formulated in the 13th UN Sustainable Development Goal in terms of reducing greenhouse gas emissions.

The world is actively researching internal processes in the body. In particular, the processes occurring in the gastrointestinal tract are currently being studied. Modern technologies allow us to create automated systems that simulate the process of assimilation of food at all its stages. These systems include SHIME and TIM [1-3]. They model the human gastrointestinal tract.

Studies of such processes in animals are only developing. By studying the body's response to a particular substance, it is possible to improve indicators related to production efficiency or product quality in poultry farms, farms, and other meat industries.

Developing a bioreactor for modeling the processes occurring in the gastrointestinal tract of chickens is accompanied by problems of maintaining the required parameters inside

* Corresponding author: dand22@bk.ru 
the bioreactor within the specified limits. The difficulty lies in the relatively small working volumes. At the same time, the requirement to reduce the volume of the bioreactor is one way to approximate the experimental conditions to the real conditions of the animal's gastrointestinal tract and reduce the carbon footprint formed during the research.

\section{Methods and materials}

The studies were carried out on a chicken gastrointestinal tract model developed by a team of researchers as part of a project «Veterinary probiotic drugs for directional modulation of animal health».

The installation is a modular design, united by a common control system based on a laptop and SCADA. (Fig. 1)

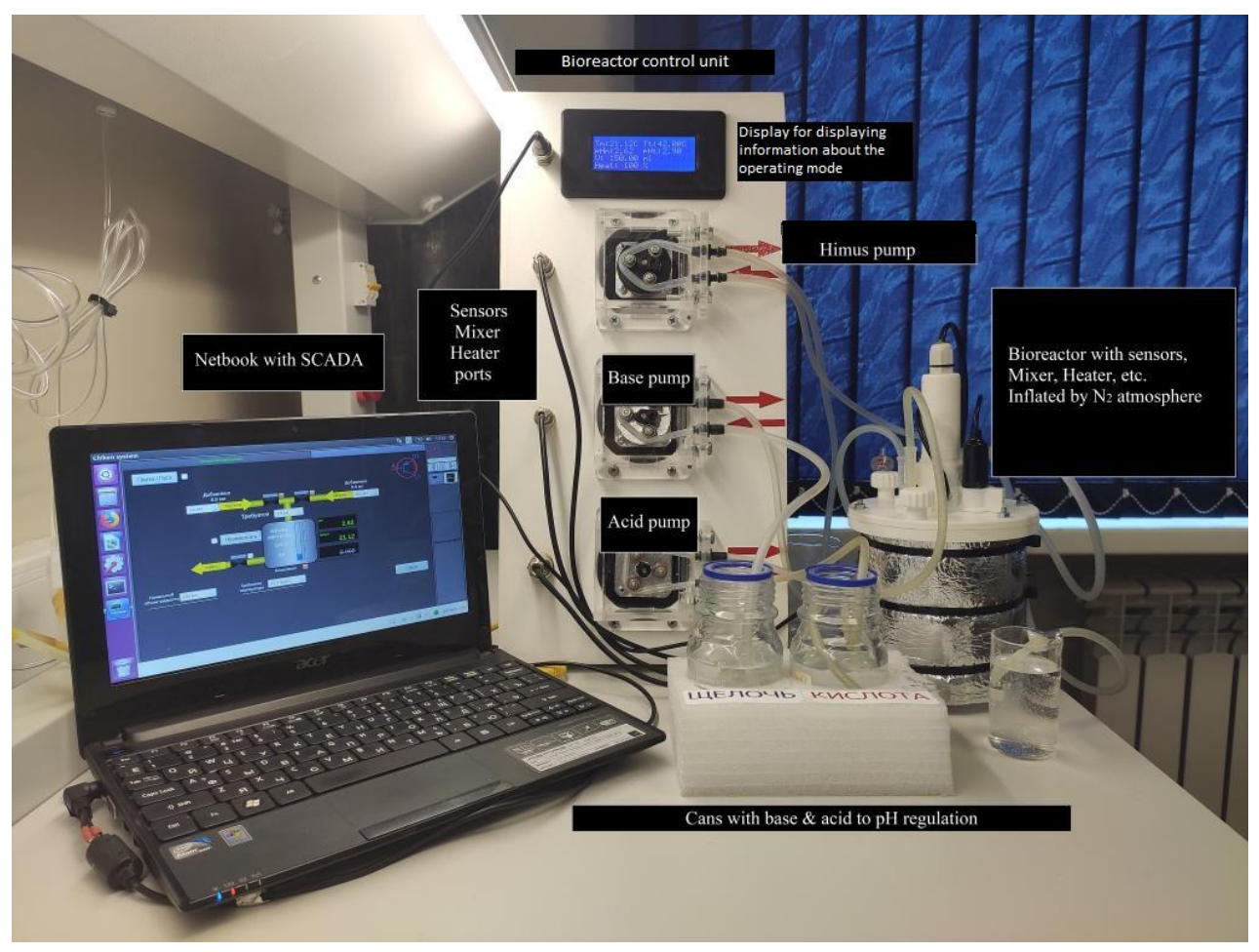

Fig. 1. Installation for modeling the gastrointestinal tract of a chicken. One module

Each module contains a microcontroller control system and provides temperature and $\mathrm{pH}$ control of one bioreactor, controlling a heater, a stirrer, and two peristaltic pumps for adding acid and alkali, and the third for pumping chime into the next reactor (Fig. 2). 


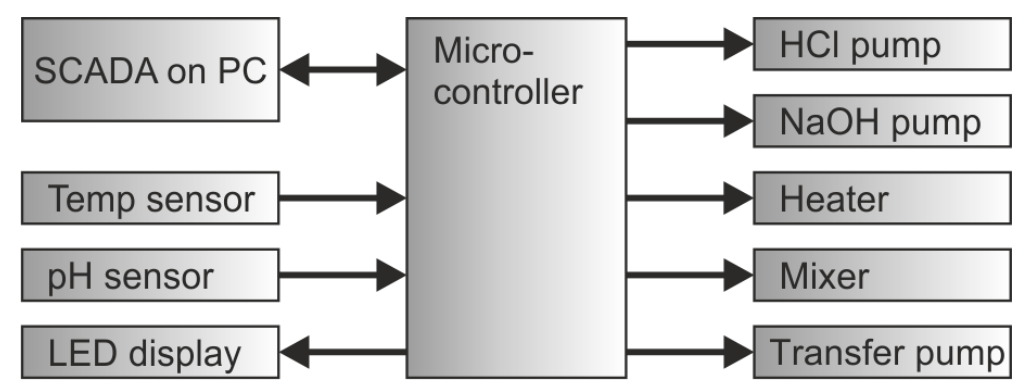

Fig 2. The structure of one cascade of GIT chicken model

The total energy consumption of the developed installation for modeling the digestive tract of a chicken consists of the following sources of consumption:

- General control system based on a mobile computer (Asus Eee PC 1015PX),

- A microprocessor-based bioreactor control system based on the Arduino MEGA 2566 microcontroller, including measuring sensors,

- Stepper motors 17HS4401 NEMA 17 peristaltic pumps,

- Electric heater for maintaining the temperature in the bioreactor,

- A drive of the bioreactor mixer based on the N20 geared electric motor.

The rated power consumption by the installation elements is given in Table 1 .

Table 1. Passport power consumed by the elements of the installation.

\begin{tabular}{|c|c|c|}
\hline & Power, W & notes \\
\hline Netbook & 10 & Normal mode \\
\hline Microcontroller & 0.8 & Normal mode \\
\hline Heater & 15 & Max power \\
\hline Mixer & 0,42 & Normal mode \\
\hline Stepper motor & 1,93 & Normal mode \\
\hline
\end{tabular}

\subsection{System features}

\subsubsection{Temperature control \& energy consumption optimization}

To control a bioreactor's temperature with a relatively small capacity is vital that the heating is carried out evenly over the entire liquid's volume. The articles $[4,5]$ describes a study of the effectiveness of three different bioreactor heating approaches in terms of energy efficiency and quality of transients. As a result of the research, it was decided to use a side heater in the chicken gastrointestinal tract modeling system.

The electric heater of the bioreactor is PWM-controlled, and the mean energy consumption for the target bioreactor temperature $42{ }^{\circ} \mathrm{C}$, and for the ambient temperature of $22{ }^{\circ} \mathrm{C}$ was about $37 \%$ of max heater power. This value was measured from the output of PWM's PID controller.

\subsection{2 $\mathrm{pH}$ measurement and regulation}

A glass electrode was used to measure the system's $\mathrm{pH}$, with the principle of operation based on measuring the potential difference [7]. For $\mathrm{pH}$ regulation used two peristaltic pumps, one for the acid $(0.1 \mathrm{M} \mathrm{HCl})$, another for the base $(0.1 \mathrm{M} \mathrm{NaOH})$. During the experiments, these pumps had short run times, mainly at the beginning of the $\mathrm{pH}$ 
normalization. The timing diagram of the $\mathrm{pH}$ regulation pump run demonstrated that uptime is less than $0.01 \%$ of experiment time.

\subsubsection{Himus transfer procedure}

Experiments, realized on considering equipment, were similar to $[8,9,11]$, adjusted for chicken metabolism [10]. According to the experiment's protocol, the fresh nutrient medium should be added to the bioreactor in a volume of $2 \mathrm{ml}$ every 5 minutes. The spent one should be pumped out in a volume of $4 \mathrm{ml}$ every 10 minutes. The capacity of the peristaltic pump is $1 \mathrm{ml}$ in $6.56 \mathrm{~s}$; that is, the topping up procedure took $13.12 \mathrm{~s}$ every $5 \mathrm{~min}$ $(300 \mathrm{~s})$, and the pumping procedure took $26.24 \mathrm{~s}$ every $10 \mathrm{~min}(600 \mathrm{~s})$.

\subsubsection{Carbon footprint evaluation method}

Estimates of the carbon footprint generated by in vivo microbiological studies on live birds and in-vitro studies performed on SHIME GIT models will be obtained.

The energy method was used to estimate the carbon footprint of the model studies. The total energy consumption during the experiment is estimated and converted into $\mathrm{CO}_{2}$ mass based on the carbon equivalent of kilowatt-hours (Table 2).

The value of the carbon equivalent of consumed electricity is known, which is $0.326 \mathrm{~kg}$ of $\mathrm{CO}_{2}$ per one $\mathrm{kWh}$. The electronic wattmeter PZEM-061, through which the whole power supply of the equipment was carried out, was used to evaluate the total power consumption. The calculation of energy consumption was carried out for the entire cycle of the experiment, lasting 48 hours. The carbon footprint of the culture medium is taken to be equal to the carbon footprint of milk (which is debatable but considered acceptable for evaluative analysis).

The chicken's carbon footprint is calculated based on a $2.5 \mathrm{~kg} 2$-month-old broiler. One experiment is carried out on one bird (the equivalent of one switching on one cascade of a GIT model reactor), after which the bird is slaughtered and disposed of.

Table 2. Passport power consumed by the elements of the installation.

\begin{tabular}{|c|c|c|}
\hline & $\mathrm{kg} \mathrm{CO}_{2}$ & notes \\
\hline electrical power, one $\mathrm{kWh}$ & 0.362 & {$[12]$} \\
\hline Milk, one liter & 1.062 & {$[13]$} \\
\hline Broiler chicken, one kg & 6.9 & {$[13]$} \\
\hline Broiler chicken, $2,5 \mathrm{~kg}$ & 10.35 & {$[13]$} \\
\hline
\end{tabular}

\section{Results and discussion}

The timing diagram of the operation of energy-consuming equipment was recorded to evaluate the carbon footprint during the microbiological experiment: a laptop with a running SCADA, a controller, a heater, a mixer, and transfer pumps. Fig. 3 represents a one-hour timing diagram of GIT model work. 


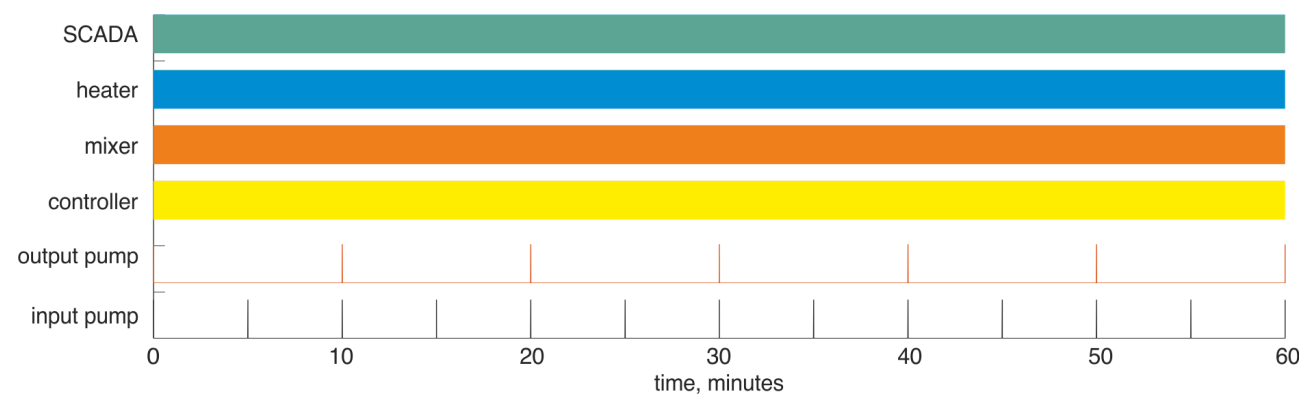

Fig 3. An one-hour timing diagram of GIT model work.

Based on the data in Table. 3, and taking into account the duty cycle of the heater, the power consumption diagram was obtained, shown in Fig. 4. Based on it, consumers can be ranked by capacity in the following order: computer with SCADA, heater, controller, mixer. The consumption of peristaltic pumps, both transfer, and $\mathrm{pH}$ maintenance systems, appears to be negligible.

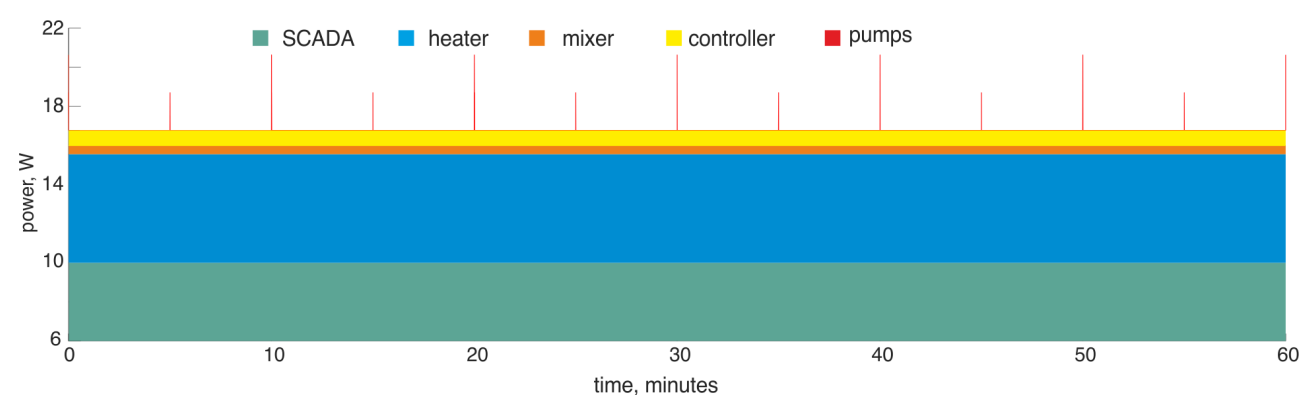

Fig 4. An one-hour power consumption distribution of GIT model work.

Processing the results in Fig. 4, and multiplying the duration of the experiment by 48 hours, it was possible to determine the consumed energy, which was $816 \mathrm{Wh}$. The energy consumption measured with an electronic wattmeter was $882 \mathrm{Wh}$, which agrees with the calculated estimate.

Converting the obtained energy values into carbon equivalent gives figures of 0.27 and $0.29 \mathrm{~kg}$ of CO2. The culture medium used in the experiment, with a volume of $\sim 1200 \mathrm{ml}$, adds 1.27 kilograms of $\mathrm{CO} 2$. Thus, the total carbon footprint of one experiment using a GIT model is, depending on the assessment method, from 1.54 to $1.56 \mathrm{~kg} \mathrm{CO} 2$

In the in vivo experiment, one broiler chicken two months old and approximately $2.5 \mathrm{~kg}$ in weight are used. The carbon footprint will be about $10.35 \mathrm{~kg}$ of CO2, taking into account the subsequent utilization.

When analyzing these results, collected in Table 3, it should be understood that they are approximate and do not take into account, for example, the carbon footprint of the infrastructure both in «in vivo» and «in vitro» experiments. However, it seems that these costs are comparable in magnitude

Table 3. The values of carbon in the cases of «in vivo» and «in vitro».

\begin{tabular}{|c|c|c|}
\hline & $\mathrm{kg} \mathrm{CO}_{2}$ & $\%$ \\
\hline «in vivo», chicken & 10.35 & 100 \\
\hline «in vitro», calculated & 1.54 & 14,9 \\
\hline «in vitro», measured & 1.56 & 15,1 \\
\hline
\end{tabular}


The results obtained allow us to draw the following conclusions:

- Experiments «in vitro», from the point of view of the formation of a carbon footprint, are about six times more environmentally friendly than experiments «in vivo» using chickens - broilers two months old,

- In experiments «in vitro», at least in the configuration used, the most significant carbon footprint $(80 \%)$ has the contribution of the nutrient medium,

- For electronic equipment (total contribution 20\%) used in in vitro experiments, the most considerable gift $(60 \%)$ is made by a computer with a SCADA system. The second most important is the heater contribution (30\%).

Based on the above provisions, the following actions can be recommended to reduce the carbon footprint in «in vitro» experiments further:

- Reducing the volume of the nutrient medium in the bioreactor,

- The transition from a laptop to single-board microcomputers such as Raspberry Pi or similar, the power consumption of which is about an order of magnitude lower,

- The use of heat-insulating jackets to reduce the energy losses of the bioreactor.

\section{Conclusion}

For the developed SHIME-style bioreactor of the chicken gastrointestinal tract, based on the data of experimental studies, an assessment and comparison was carried out carbon footprint value between «in vivo» and «in vitro» microbiological experiments. For «in vitro» estimation - use analysis and results of experiments, carrying out on developed chicken GIT model. For « in vivo» estimation, use the carbon footprint of two mounts old broiler chicken. Analyses showed that «in vitro» carbon footprint constitutes about $15 \%$ of «in vivo» one. The most significant contributors to «in vitro» carbon footprint are nutrient medium $(80 \%)$, then control computer $(10 \%)$, then heater $(5 \%)$. Recommendations for further carbon footprint reduction for «in vitro» experiments are formulated.

The research was supported by the grant «Veterinary probiotic drugs for directional modulation of animal health» №075-15-2019-1880, 03.12.2019, of the Russian Ministry of Science \& Higher Education.

The authors are grateful to professor M.L. Chikindas for the common project guideline and dr. A.D.Lukyanov for the management of the engineering part of the project.

\section{References}

1. Van de Wiele T., Van den Abbeele P., Ossieur W., Possemiers S., Marzorati M., The Simulator of the Human Intestinal Microbial Ecosystem (SHIME®). In: Verhoeckx K. et al. (eds) The Impact of Food Bioactives on Health. Springer, Cham (2015)

2. Pieter Van den Abbeele, Koen Venema, Tom Van de Wiele, Willy Verstraete, Sam Possemiers, Different Human Gut Models Reveal the Distinct Fermentation Patterns of Arabinoxylan versus Inulin, J. Agric. Food Chem., 61 (2013)

3. Chiara Anna Maria Fois, Thi Yen Loan Le, Aaron Schindeler, Sina Naficy, Dale David McClure, Mark Norman Read, Peter Valtchev, Ali Khademhosseini, Fariba Dehghani, Models of the Gut for Analyzing the Impact of Food and Drugs, Adv. Healthcare Mater, 8 (2019)

4. V. A. Chistyakov, A. D. Lukyanov, S. V. Chapek, D. Yu. Donskoy, O. I. Katin, IOP Conf. Series: Materials Science and Engineering 900 (2020)

5. Lukyanov, Alexandr \& Donskoy, Danila \& Bykador, Vitaly \& Chuveyko, Mikhail \& Kasyanenko, Elena, E3S Web of Conferences. 104 (2019) 
6. H. Torres, R. O. Cristancho, Implementation of the primary $\mathrm{pH}$-measurement system and its uncertainty estimation, J. Phys.: Conf. Ser. 1119012011 (2018)

7. Koen Venema. The TNO In Vitro Model of the Colon (TIM-2), K. Verhoeckx et al. (eds.), The Impact of Food Bio-Actives on Gut Health, p.293-304 (2015)

8. Van den Abbeele P, Roos S, Eeckhaut V, MacKenzie DA, Derde M, Verstraete W, Marzorati M, Possemiers S, Vanhoecke B, Van Immerseel F, Van de Wiele T. Microb Biotechnol, 106-15 (2012)

9. Giuliani, Camilla and Marzorati, Massimo and Innocenti, Marzia and Vilchez-Vargas, Ramiro and Vital, Marius and Pieper, Dietmar H. and Van de Wiele, Tom and Mulinacci, Nadia, Dietary supplement based on stilbenes: a focus on gut microbial metabolism by the in vitro simulator M-SHIME ${ }^{\circledR}$, Food Funct, p 4564-4575 (2016)

10. Stanley, D., Hughes, R. J., \& Moore, R. J., Applied microbiology and biotechnology, 98(10) (2014)

11. Korostylev, N., Prazdnova, E., Mazanko, M., Chistyakov, A., Zharov, V. Genetic basis of the probiotic properties of Lactobacillus, Ecological and Biological Well-Being of Flora and Fauna (EBWFF-2020), E3S Web of Conferences, 203 (2020)

12. How much carbon dioxide is produced per kilowatt hour of U.S. electricity generation?, U.S. Energy Information Administration, URL: https://www.eia.gov/tools/faqs/faq.php?id=74 (2019)

13. Environmental Working Group Meat Eaters Guide: Methodology 2011, Environmental Working Group, 65 p, URL: https://static.ewg.org/reports/2011/meateaters/pdf/ methodology_ewg_meat_eaters_guide_to_health_and_climate_2011.pdf (2011) 